\title{
Controlled outflow of storm water without flooding
}

\author{
Karel Adámek ${ }^{1, *}$, Jan Koláŕ, and Pavel Peukert \\ ${ }^{1}$ VUTS a.s., Dept. of numerical simulations, Svárovská 619, 46001 Liberec, Czech Republic
}

\begin{abstract}
There are many types of devices used for various purposes, called as vortex valves. The aim of this paper is the design of vortex valves, determined for controlled higher outflows from retention tanks. The paper follows the previous study of smaller sizes of vortex valves. The method of flow numerical simulation allows us to identify the reason of the twobranch operational (resistance) characteristic of the solved valves and the suitable sizes of the main valve dimensions for the given flow rate and water level.
\end{abstract}

\section{Introduction}

There are many types of devices, used for different purposes, called vortex valves. The aim of this paper is the design of vortex valves, destined for controlled higher outflows from retention reservoirs. The principle of valve activity is described curiously by $\mathrm{Mr}$. Google and many dealers and producers, for instance, as "the effect is given by flow phenomenon" or "it works on the principle of hydraulic phenomenon". Reality is not so mysterious, the flow inside of such a valve is possible to describe by numerical flow simulation, completed by some essential equations of fluid mechanics, see [1-7] etc.

\subsection{The previous model}

In the previous work [7], the analysis and solution of vortex valves were made for lower flow rates, therefore, here, it should be repeated the main results, partially used for higher flow rates.

Typical resistance characteristic $\Delta \mathrm{p}(\mathrm{Pa})=\mathrm{f}(\mathrm{m}(\mathrm{kg} / \mathrm{s}))$ of the solved vortex valves is composed from two branches of different gradients and separated by an area of some discontinuity / instability. Typical results of unified dimensions for low and high mass flows are presented in Par. 4.6 below for several combinations of both inlet (tangential) and outlet (axial) orifices. The reason of the two-branch characteristic is explained in [7], too. The preliminary design for higher flow rates is realized as an enlargement of cross sections from the verified solution [7], proportional to the higher mass flow. This hypothesis should be verified on a specific design using the assortment of semi-finished products and of actual vortex valves [8-11]. At first, it will be necessary to enlarge the valve body, to insert the

\footnotetext{
* Corresponding author: karel.adamek@vuts.cz
} 
enlarged inlet tube. Due to the unified design, it should be suitable to use the same valve body and the mass flow to adjust them by replaceable outlet inserts. As found out in [7], the branch of higher gradient is valid when the valve body is full by water, and the transition between the branches arrives when the water level is on the upper outline of the valve body, approx. For lower water levels, the valve body is not full with water and the inner rotation of water does not arise. It exists a free water level, only, waved by the tangential inlet from below and water is then freely flowing out through the axial outlet without spraying.

It is possible to await that in a larger valve body, the transition between the branches of characteristic begins for a higher pressure than in [7]. However, here assigned and used pressure gradients are similar as in [7], therefore, it is possible to await that the real valve operation could be in the unstable transitional region. In addition, it is necessary to check the global adequacy of the new dimensions.

Note: The aim of this vortex valve solution in not any perfect spraying of outflowing liquid, as for instance in the spraying gun, but the only decreasing of the dynamic effect of the outflowing liquid on the surroundings. The aim is reached for smaller pressures without sprayed outflow, too, with free water level inside the valve body - some part of the flow energy is changed into flow losses inside the valve body (inlet, friction in the inlet tube and during the rotation, the change of the flow direction from peripheral into the axial, flow spraying, back flow of the air from the surrounding, etc.).

Designing the valve, it is necessary to fulfil two contradictory requirements: For a valve of high volume, flow larger inlet and outlet diameters should be designed together with the larger valve body diameter, too. But by this way, the peripheral velocity wo is decreasing and after its second power the centrifugal acceleration

$$
a o=w o^{2} / r
$$

is decreasing, too. And more, through a small volume of the valve body, it cannot flow the required high water flow.

Here, it is alternatively designed a simple prismatic shape of the valve body, where the smaller diameter ensures a quite high peripheral velocity and centrifugal acceleration, too, and the necessary flow cross section is designed in the axial direction, depending on the necessary flow volume (see for instance the Par. 2.3 below).

\section{Flow numerical simulation}

At first, the above given hypothesis should be verified, i.e. to derive the shape of the new valve for higher flows by a simple increasing of main dimensions from the verified solution [7]. The procedure of the model preparation and solution is identical with [7], therefore, only a short description is made here.

\section{a. Model description}

Geometry - as in [7], adapted proportionally to the prescribed flow.

Mesh - model dimensions are various in the range over than 1:100, so in rough mesh some inaccuracy should be taken into account. Never mind, such a possible error is identical for all solved cases and comparable results of many solved cases are obtained quickly and reliably.

Boundary conditions - inlet hydrostatic pressure up to $2.0[\mathrm{kPa}]$ of the phase 1 (water) is defined in the center of the inlet tangential tube, outlet in the atmosphere $0 \mathrm{kPa}$ is defined on the surfaces of the surroundings, added along the axis of the valve body. In the outlet, there is defined backflow of the phase 2 (air from the surroundings). At the beginning, in all outlet volumes, it is defined phase 2 (air), only, and inside the valve, the phase 1 (water), only.

Note: The boundary condition "inlet pressure" is simply defined as constant in the whole inlet cross section, i.e. as hydrostatic pressure of water column between the water level and the center of inlet. The real difference between the highest and lowest point of the inlet cross 
section in [7] is typically $\pm 0.25 \mathrm{kPa}$ - it is neglected here, to simplify the model, without the next connecting channel between the valve inlet and the surrounding free water level. Really measured differences between the simulated and real flows [8] are less than $5 \%$ approx.

Solver: Turbulence model k- $\omega$ SST. Used stationary two-phase model "mixture", the unsteady model VOF (volume of flow) needs a much longer solution time, but here it is not necessary to solve details of the flow field [7]. The solution is regarded as converged, when the difference between the inlet and outlet flow is less than $0.1 \mathrm{~kg} / \mathrm{s}$.

Results - during several iterative steps, the designed main dimensions are changed to get flow as defined in advance. Illustrative figures of the flow fields are in the text, as necessary. For the text shortening, some scales are left out - in such case, it is in force that red = maximum value and blue $=$ minimum value, identically with the wave lengths in the spectrum of visible light. For a qualitative comparison of individual cases, exact scales are not necessary.

\section{b. Trial prismatic model}

The results of this trial valve are added as an illustration, only. Contrary to the next cases, its axis is vertical with outflow up, therefore without a disturbing effect of gravity, as discussed in [7]. Similar case [5] presents the vertical axis with outflow down, when the upper surface of the valve remains dry. With an increasing of the mass flow, the valve main dimensions must be increased, too (body, inlet, and outlet). With increasing the body diameter, the centrifugal acceleration, necessary for a good water spraying, is decreasing with the second power of the body diameter. Therefore, the vaulted valve bodies [7] using semifinished products [8-11] are not suitable because their outlines are too large. A better solution is a prismatic valve body, where the inlet size is simply changed by a change of the body width (by the cylinder height, in the axial direction) and at a suitable body diameter, the velocity and flow along the periphery do not decrease under the suitable limits. For a given pressure gradient of $16 \mathrm{kPa}(1.6 \mathrm{~m})$, the ideal outflow velocity is

$$
\text { wid }=\sqrt{ }(2 . g . H)=\sqrt{ }(2.9 .81 .1 .6)=5.6 \mathrm{~m} / \mathrm{s}
$$

and the theoretic flow of $143.43 \mathrm{~kg} / \mathrm{s}$ through the prismatic inlet of $0.16 \times 0.16 \mathrm{~m}$. The inlet flow from the simulation is $61.82 \mathrm{~kg} / \mathrm{s}$, only, so the flow coefficient is 0.43 (the ratio of « real », here simulated, flow to the ideal one). The next results of the simulation are the air suction of $1.02 \mathrm{~kg} / \mathrm{s}$ through the front face of the surroundings and the outlet flow of $62.77 \mathrm{~kg} / \mathrm{s}$ through the cylindrical periphery of the surroundings. Therefore, the error of the mass balance is $0.07 \mathrm{~kg} / \mathrm{s}$, i.e. $0.1 \%$ of the inlet flow. It is negligible, therefore, the simulation was finished and evaluated.

For a presentation of the complicated flow field, Figure 1 to Figure 4 present several images of flow field parameters. For a better resolution, different scales are used for the same parameter in different cross sections.
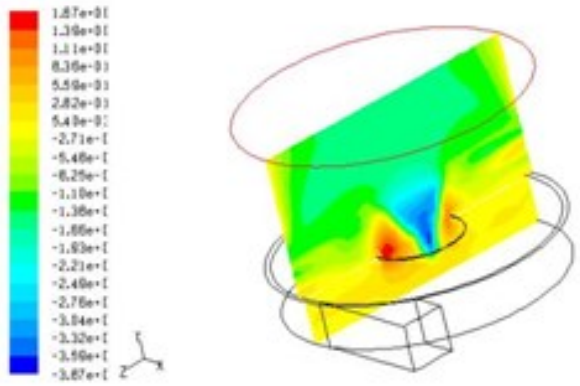

Axial velocity (outflow up along the orifice outline, suction down along axis)
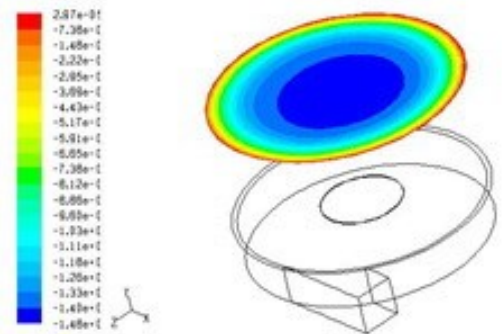

Axial velocity at outlet cross section

Fig. 1. Axial component of velocity. 
Figure 1 presents the highest value of the axial component of velocity at the outlet edges, the highest suction inside along the outlet axis. In the area opposite to the tangential inlet, the flow is higher than at the side close to the inlet. At the outlet, the backflow is visible along the axis.
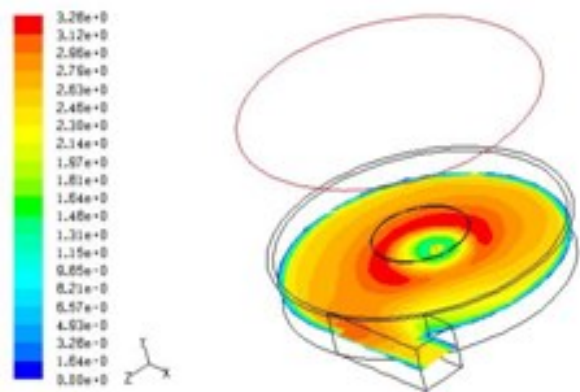

Velocity at inlet plane

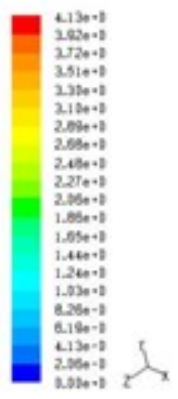

Outflow velocity at the periphery of the area

Fig. 2. Inlet and outflow velocity.

Figure 2 left presents the absolute value of the velocity vector in the cross section through the inlet axis. Here, it is approximately evaluated the peripheral velocity of the rotating liquid body as $2.5 \mathrm{~m} / \mathrm{s}$ at the radius of $0.4 \mathrm{~m}$. In this estimation, some error is expected - it is hardly to estimate, what part of the rotating liquid body to use for the determination of average peripheral velocity and what is the average radius of such a body. Nevertheless, from such an estimation, the centrifugal acceleration is $15.63 \mathrm{~m} / \mathrm{s}^{2}$. Due to the inaccuracy at determination of peripheral velocity and flow radius and also that the gravity affects here in across direction, it supposes that a perfect spraying of the flow will not be created, rather the flow will be spilled out along the whole outlet periphery with some irregularity, due to the one-sided inlet. At the periphery of the outer area (the surroundings, Figure 2 right, there is observable some flow thickness, which is flowing on the body surface out in the direction across to the axis, the most intensive flow is opposite to the inlet. Along the periphery, there are visible some irregularities, feeble points, etc. It could be the error of the simulation due to the coarse mesh or little area of the surroundings - so that to get a shorter time of solution for many solved cases. Similarly, at the upper edge, there are some irregularities, but absolutely small, here, it is hardly any flow.
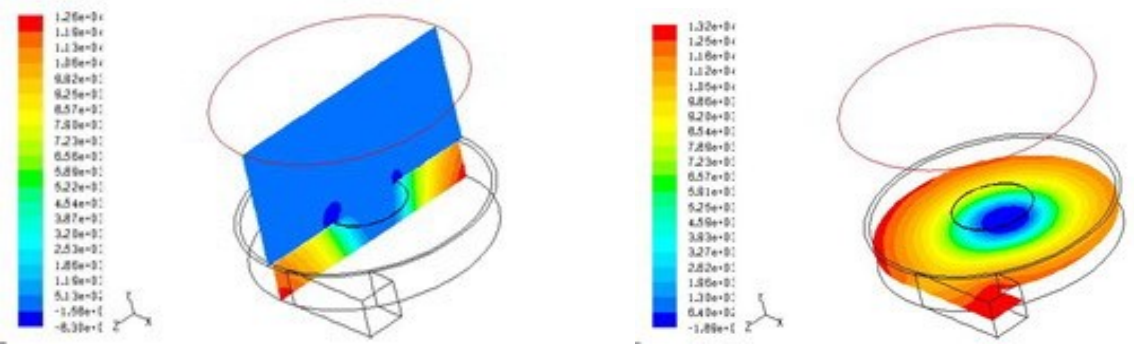

Fig. 3. Pressure - inversed to the velocity (max. in the inlet, min. in the outlet).

Pressure is inverse to the velocity (Figure 3), maximum at the periphery of the valve body, it is decreasing to the axial outlet, where it is going under the atmospheric pressure of the surroundings. 

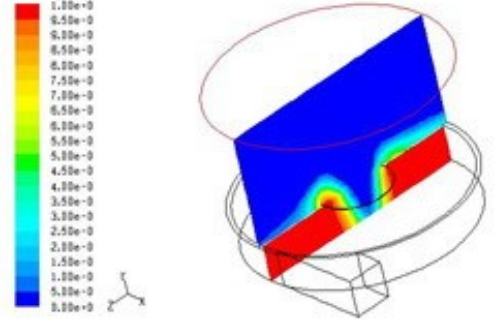

Axial section $($ water $=$ red, $\max$. inside $)$
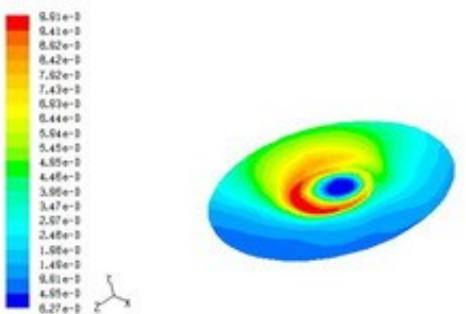

Outlet plane - asym. spraying

Fig. 4. Velocity field of water phase.

The field of the water part (phase 1) in Figure 4 presents the water spillage on the body surface after the outflow. The outflow is not symmetrical, facing to the tangential inlet, there is the higher peripheral outlet. The same is visible in the cross section in the outlet plane, too.

\section{Modelled solutions}

From Par. 2.2, it is visible that the detailed presentation of all flow field parameters of each solved case is very extensive, therefore, here are presented directional fields of both phases (water and air), only, at the outlet from the cylindrical surroundings - through both front and peripheral surfaces. They indicate the quality of the sprayed flow. From the results of four solved cases here are demonstrated two of them, only.

For the case $1(21.3 \mathrm{kPa}, 30 \mathrm{~kg} / \mathrm{s})$, the assigned pressure is quite high, therefore, the spraying is very good. In the smaller valve body and at the higher pressure gradient, the image of the outlet directional field is very good, see Figure 5, the deviation of the individual vectors from the radial direction is the consequence of the one-sided tangential inlet, followed by flow rotation.

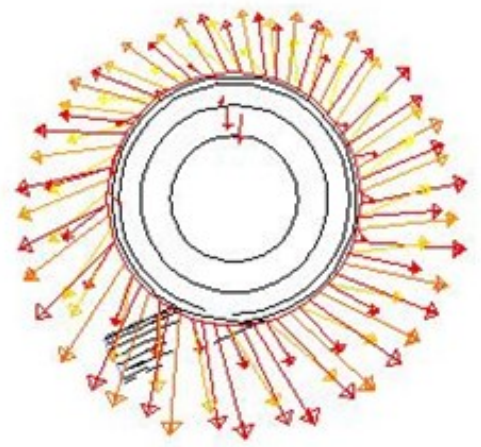

a) phase 1 -water is going out through the periphery, only, not through the central part

Fig. 5. Directional fields at the outlet side - case 1.

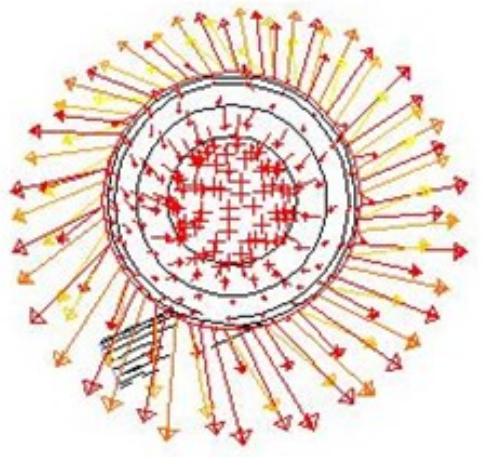

b) phase 2-air is drawn in through the axial face, and is going out through the periphery, together with water

A large prismatic arrangement of the case 2 with a relative small pressure gradient $(15.9 \mathrm{kPa}, 105 \mathrm{~kg} / \mathrm{s})$. The result in Figure 6 shows the partial spraying, only, through the upper part of the periphery and air suction through both the axial middle part and lower peripheral part. 


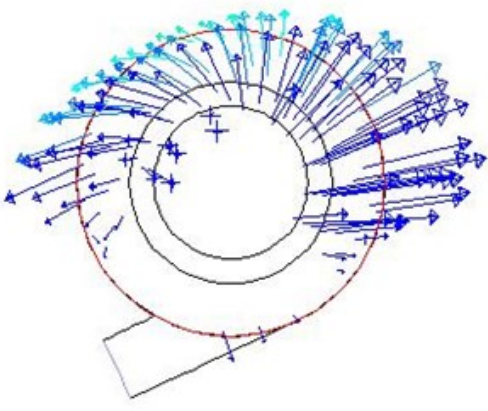

a) phase 1 - water is flowing through the upper part of the periphery, only, it is visible small backflow

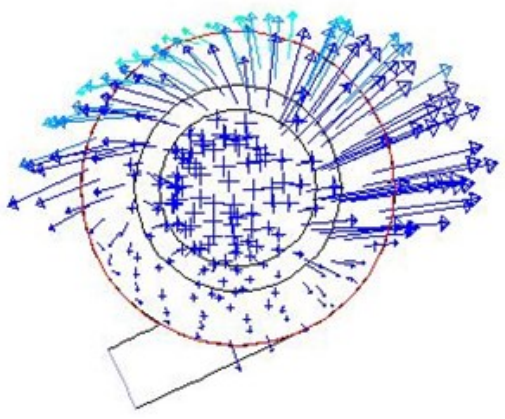

b) phase 2 - air is drawn inside through both middle axial part and lower part of the periphery, and going out together with water through the upper part of the periphery

Fig. 6. Directional fields at the outlet side - case 2 .

Operating point of the next case $3(16.3 \mathrm{kPa}, 22 \mathrm{~kg} / \mathrm{s})$ seems to be similar to the case 1 , but the spraying is bad, due to the low pressure - therefore, without Fig. The only remedy is the decreasing of the valve body diameter with the original sizes of the rest parts. By this, the velocity is increasing and the spraying will be better. But the outer diameter decreasing is not possible due to "short-circuit" flow from inlet into outlet, without flow rotation.

The biggest case 4 of prismatic inlet $0.16 \times 0.32 \mathrm{~m}(15.8 \mathrm{kPa}, 125 \mathrm{~kg} / \mathrm{s})$. The simulated mass flow corresponds to the defined one, outlet spraying is good, therefore, without next Fig.

Note: The case 4 above was modified by the spiral outer case (designed without detailed calculations). Comparing the results of both cases, it is possible to state that the resulting directional field of the spiral valve body is similar to the cylindrical one. It is interesting that the flow is increased of $20 \%$ approx., at the same boundary conditions. It means that the resistance of such a valve is lower, but it is not good for the intended purpose - to suppress the dynamic effect of the outflow on the surrounding.

\section{Conclusions}

\section{a. Geometry}

For each of 4 solved cases, suitable main geometric parameters of the valve (outlines, inlet and outlet diameters) were found to get the prescribed water flow for a prescribed pressure gradient (hydrostatic pressure of the given water level).

The initial dimensions of the new cases were derived from the former solved and verified cases, using simply the continuity equation on the flow change by a cross section change is the initial inlet parameter, only. But flow resistances during the complicated 3D flow (inlet, rotation, interaction of rotating and entering flow, transition from rotation into axial outlet, spraying, friction, etc.) have important influence. For such case, it is not possible to apply simple formulas from the basic course of fluid mechanics, but a numerical simulation of 3D flow is necessary.

The modelled solution should be verified by experiment - each model is some approximation of the reality, only, it is necessary to check if any simplification does not change the results too much.

The presented result of each solved implicit case is reached in several iterative steps. From the convergence course of the solution, it is possible to estimate that some cases with 
oscillating convergence could be unstable in real operation, i.e. with irregular spraying, pulsating flow, higher noise level, etc.

The main aim of the solution is not the quality of the spraying at the outlet, but a reduction of the dynamic effects of the outgoing flow, therefore, next simulations were not realized. In the given cases, the water level is relative low, so at relative large inlet and outlet it is not to expect uniform spraying along the whole outlet periphery. The initial design uses the vaulted shape of the valve body, for higher flows it is used a flat design, where the cross section is changed by the body width, not by body diameter.

\section{b. Spraying quality}

Influence of the outlet edge on the spray quality was tested on the case 4, only, see Figure 1 above. The best solution is given by the thin and sharp outlet edge, but the long cylindrical extension damps the spraying effect. Some improvement of it is given by the chamfered outer edge, where the flow adheres to the chamfering and dissipates in the radial direction. The effect is known from the so-called wall nozzles - for suitably designed dimensions, the outflow can be adhered of over $180^{\circ}$, see [12-13], etc.

\section{c. Flow coefficient}

The so-called flow coefficient of individual cases is evaluated as a check value and as an information item about the flow quality. It is defined as the rate of real (here simulated) flow and that of idealized flow. As an information item, the coefficient for the so-called quarter circle nozzle (well rounded) reaches a value of $0.95-0.97$, for the simply drilled orifice in the wall a value of 0.64 , for smaller vortex valves solved in [7] a value of 0.28 , only. Here solved smaller and vaulted cases $1+3$ have the coefficient of $0.50-0.58$, larger and flat cases $2+4$ that of $0.37-0.45$. Higher influence on the flow quality has the vaulted shape, the influence of larger dimensions is not so important here.

\section{d. Basic directives of the valve design}

In a smaller valve body, the peripheral velocity is higher, so the spraying is better, too. But the inlet diameter must pass between the outlines of the valve body and the valve axial outlet to prevent short-circuit flow between the inlet and the outlet without rotation.

Both inlet and outlet diameters define approximately the possible flow range. If the outlet is smaller than the inlet, the flow tends to be straight and strong without spraying. If the outlet is equal or larger than the inlet, the water spraying will be good.

\section{e. Error estimation}

Hydrostatic pressure in the inlet of the models is simply defined as constant, but really there is some pressure difference of $0.35 \mathrm{kPa}$, approx., between the lowest and the highest point of the inlet cross section - in simulations [7] neglected. The difference between the simulated and the really measured flow is not more than $5 \%$ approx. [7], due to slightly waved both inlet and outlet real water levels.

\section{f. Operational characteristics}

Inlet data for a valve designer are not only the main dimensions, but the so-called operational (resistance) characteristic $\Delta \mathrm{p}=\mathrm{f}(\mathrm{m})$, too, well known as a quadratic function $\Delta \mathrm{p}=\mathrm{f}\left(\mathrm{m}, \mathrm{m}^{2}\right)$. 
In those cases 1-2-3-4 are solved high flows, only, just before the overflow over the security overfall. The lower branch of the characteristic is not solved here. The results are compared with the former results [7], see Figure 7.

For the valve body diameter of $780 \mathrm{~mm}$ (cases 2+4), it is possible to estimate that the transition between the lower and the upper branch of the characteristic is of $10 \mathrm{kPa}$ approx. Therefore, simulations were made for the range of $10-16 \mathrm{kPa}$, also for valves of medium sizes (cases 1+3), where the transition area could be estimated under $10 \mathrm{kPa}$. Four solved points of each characteristic are used as the minimum for quadratic functions.

As found out in [7], the higher branch of the characteristic begins when the valve body is full of water. For here solved higher flows, i.e. larger valve bodies at the same maximum water level it could mean the operation in transitional area between both branches. So the spraying quality could not be good, but the main task of the observed valves is to suppress the dynamic effect of the flow, not the spraying quality.

All new characteristics, as a result of many simulated cases, are presented in the common graph in Figure 7, checked with the characteristics of small valves [7] (their inlet/outlet = $80 / 80-200 \mathrm{~mm}$ ). Due to a large extent of flows, the scale of the x-axis is logarithmic. The graph confirms the preliminary estimations that the used lower point of the characteristic of $10 \mathrm{kPa}$ is far from the transitional area for cases $1+3$ (middle flows, middle valve sizes), but for cases $2+4$ (large dimensions, large flows), the lower parts of their characteristics are bent, which signs the vicinity of the transitional area.

Note: For cases of small flows, solved in [7], the transitional area is of 3-4 $\mathrm{kPa}$, only.

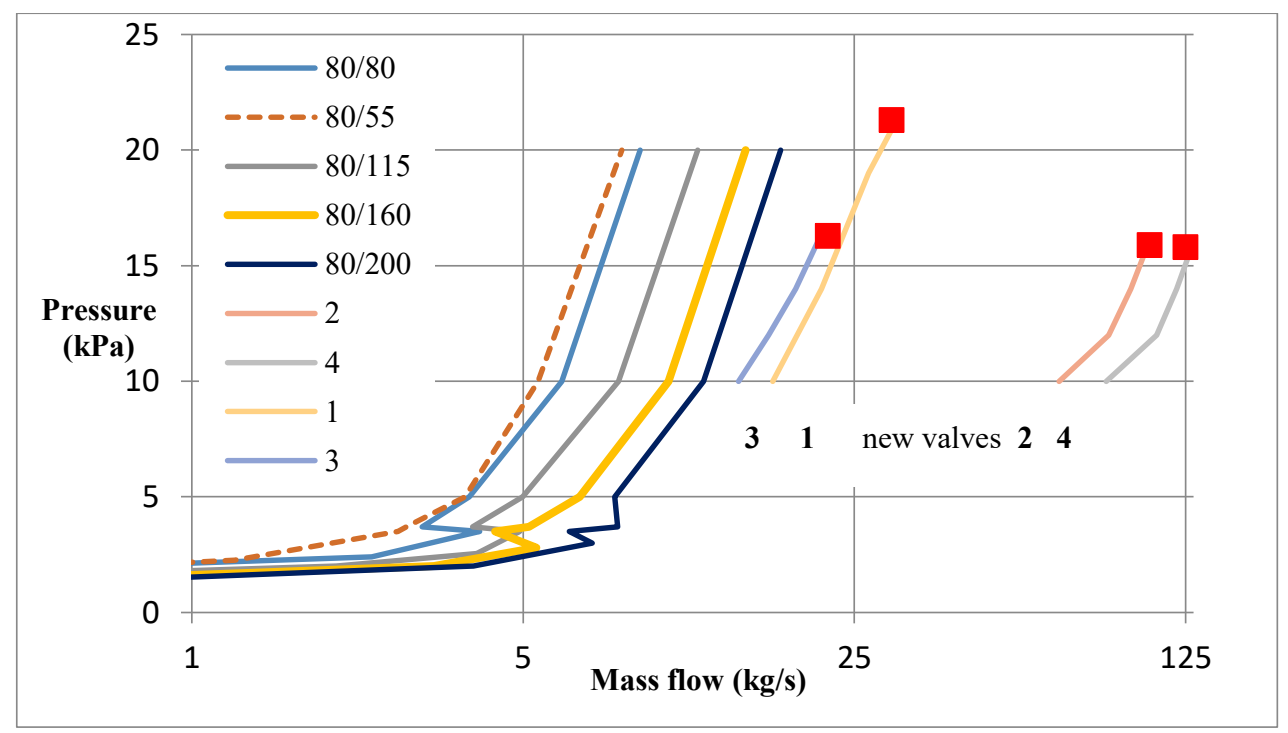

Fig. 7. Operational (resistance) characteristics of solved valves 1-2-3-4, together with valves [7].

Our acknowledgment is given to VUTS Liberec - Center for Development in Machinery Research for the support in the framework of the grant NPU-LO1213, granted by the Czech Ministry of Education, Youth and Sport.

\section{References}

1. Vortex valve, patent US 3219048 from 23.11.1965

2. D. Jarman, D. Butler, G. Tabor, R. Andoh, ICE proceedings - Engineering and computational mechanics, 168, issue EM1 
3. R. Andoh, M. Faram, K. Osey, Proc. of the 6th int. water sensitive urban design conference and hydropolis, Perth Australia (2009)

4. H. Steinriede, M. Merschdorf, Wirbeldrossel - Produkt information, UFT 2016

5. H. Brombach, H. Steinriede, Vertical vortex valve - product information, UFT 2014

6. V. Tesař, Superquadratic fluidic restrictors and their applications, Advances in fluid mechanics IX, WIT Transactions on Engineering Sciences, 74 (2012)

7. K. Adámek, J. Koláŕ, P. Peukert, Vortex valve, EFM Mikulov (2017)

8. Catalogue of semi-finished parts www.zyksal.cz

9. Catalogue of valves producer www.bmto.cz

10. DIN 28011 - Vaulted foreheads, stainless steel

11. DIN 17458 - Tubes seamless, stainless steel

12. H. Coanda, US Patent No. 2,052,869. Device for Deflecting a Stream of Elastic Fluid Projected into an Elastic Fluid (1936)

13. K. Adámek et. al, Wall flows and their applications, in: Int. Journal of Mechanical Engineering and Applications, special issue Numerical Flow simulations in textile technology, 3(2), 28-32 (2015) 